\title{
La Historia de dos Ciudades: Procedimientos Paralelos y Reformas al Arbitraje de Inversión
}

\author{
Por Ignacio Torterola* y Damasia Escudero**
}

Recibido/Received: 31/01/2016

Aceptado/Accepted: 19/08/2016

SUMARIO: 1. Introducción. 2. Procedimientos Paralelos en Ecuador: Los Arbitrajes Burlington y Perenco. 3. La Fisonomía de los Procedimientos Paralelos. 4. Conclusión.

PALABRAS CLAVE: arbitraje de inversiones, Burlington, Perenco, reforma, tratado de inversiones.

KEYWORDS: amendments, Burlington, investment arbitration, investment treaty, Perenco.

RESUMEN: El sistema arbitral de inversiones ha recibido severas críticas en los últimos años, y se ha manifestado que una reforma debería hacerse de inmediato. En este sentido, las decisiones de los casos Perenco y Burlington confirman lo

* Socio en la práctica de Arbitraje Internacional y Litigios Internacionales del Estudio Jurídico GST LLP. Ex miembro (por concurso) del Servicio Exterior de la Nación Argentina, Sub-Coordinador de la Dirección de Asuntos Internacionales de la Procuración del Tesoro de la Nación Argentina y representante de Argentina ante el CIADI. Representante de Argentina ante el Grupo de Trabajo II de la CNUDMI sobre Arbitraje Internacional, el Comité de Inversiones de la OCDE y forma parte del Grupo de Expertos en Arbitraje de Inversión de la UNCTAD y del London Center for the International Law Practice (LCILP). Árbitro en el Centro de Arbitraje de la Cámara de Comercio de Quito, y en los Centros de Arbitraje de la AMCHAM Lima y la Cámara de Comercio de Lima.

** Ex Asociada del Estudio Marval O'Farrell \& Mairal, Argentina. Investigación \& Arbitraje, Washington DC. LL.M en Fordham University, New York.

D. Escudero e I. Torterola, "La Historia de dos Ciudades: Procedimientos Paralelos y Reformas al Arbitraje de Inversión”, Revista Ecuatoriana de Arbitraje, No. 7, 2015. 
dicho, toda vez que debido a los procedimientos paralelos en ambos casos y la autonomía con la que los tribunales actuaron las decisiones fueron distintas e incluso contradictorias. Es así que se evidencian las consecuencias negativas que este escenario acarrea en el ya cuestionado sistema de arbitraje de inversiones.

ABSTRACT: The investment arbitration mechanism has been criticized in the last years for many reasons; consequently, the arbitration community has suggested several amendments in order to improve the system. The decisions in cases Perenco and Burlington confirmed the critics investment arbitration mechanism has been receiving because of the contradictory decisions the tribunals made due to the parallel processes and autonomy they had. In this context, these cases show the negative consequences and the importance of upgrade the system.

\section{INTRODUCCIÓN}

El presente trabajo surge a partir de una invitación del Instituto Ecuatoriano del Arbitraje, pero también refleja una reciente charla que uno de los autores presentará en ocasión del III Seminario de Derecho Público y Derecho Internacional (2015), organizado por la Procuraduría General del Ecuador. Dado que el presente número del Anuario Ecuatoriano se refiere precisamente a las Reformas al Sistema de Arbitraje de Inversión, la coincidencia no pudo haber sido más feliz, especialmente cuando hacemos uso de la casuística ecuatoriana para ejemplificar la posición que mantenemos a través de este artículo de opinión y que en otras oportunidades hemos explicado a través de la experiencia de otros Soberanos.

Los organizadores del III Seminario de Derecho Público y Derecho Internacional (antes referido) pusieron en mis manos un muy buen artículo de opinión escrito por Bernardo CREMADES e 
Ignacio MADALENA sobre los Procedimientos Paralelos en el arbitraje internacional ${ }^{1}$. Sin embargo, como explicaré en las conclusiones, en el caso de los Arbitrajes de Inversión, el fenómeno de los Procedimientos Paralelos van más allá de la implicancia que puede tener la cuestión desde un punto de vista procesal, pues plantean y constituyen un cuestionamiento vivo al sistema arbitral de inversiones, sistema que requiere reformas urgentes para evitar procedimientos paralelos, y lo que es más grave, las decisiones contradictorias que se dictan a partir de ellos.

Analizaremos dicha problemática a partir de dos casos concretos iniciados contra la República del Ecuador, pero que parten del mismo nodo factual: Burlington Resources Inc. v. La República de Ecuador ("Caso Burlington" o "Burlington") y Perenco Ecuador Ltd. v. La República de Ecuador y Empresa Estatal Petróleos del Ecuador ("Caso Perenco" o "Perenco). Debido a su casi idéntica matriz fáctica y a las decisiones diametralmente opuestas a la que arribaron los Tribunales arbitrales, constituyen un planteo vivo para que los operadores judiciales y arbitrales, así como el legislador ecuatoriano, se planteen si este modo de resolver controversias es aquel adecuado para brindar justicia en controversias donde se encuentra afectado el erario público.

\section{Procedimientos Paralelos en Ecuador: Los Arbitrajes Burlington y Perenco}

La historia de la explotación de los recursos naturales y, especialmente aquella de la explotación del gas y del petróleo, es una historia viva en el Ecuador, no sólo por ser un país que en gran parte de su historia ha dependido fuertemente de los recursos naturales (como es en general la historia de los países latinoamericanos), sino además porque la explotación de estos recursos ha impactado en la sociedad ecuatoriana misma, en su relación con el medioambiente y en la coexistencia con los pueblos originarios.

1. B. Cremades e I. Madalena, "Procedimientos Paralelos en el Arbitraje Internacional", Revista Peruana de Arbitraje, No. 6, 2008. 
Sin embargo, la historia del Ecuador y su relación con el petróleo y el gas, no es diferente a aquella de tantos otros países que descubrieran que bajo su corteza terrestre yacen grandes cantidades de este oro negro, pues los Estados constantemente cambian el marco contractual y regulatorio con el objetivo de poder retener una parte de las ganancias que la empresa privada obtiene a través de la explotación de estos recursos no renovables. Esta es una tendencia mundial y una práctica no sólo de las economías en desarrollo, sino también de las naciones desarrolladas ${ }^{2}$. Ecuador no fue una excepción al respecto.

En relación a ello, el sustrato fáctico de los arbitrajes en comento se inserta precisamente en el incremento extraordinario de los precios del petróleo a partir de los primeros años del nuevo siglo y en los intentos del Estado ecuatoriano para incrementar su participación en las ganancias extraordinarias de los contratistas en los Contratos de Participación. Hagamos un poco de historia:

Hasta los años 90s la figura contractual prevaleciente para la explotación de hidrocarburos en Ecuador era aquella del Contrato de Prestación de Servicios. Bajo esta figura contractual, el gobierno era el único propietario del petróleo y al inversor se le pagaba una tarifa para que prestara servicios de exploración y explotación del recurso. En el año 1993, y con el cambio de paradigma ideológico introducido con las nuevas ideas liberales que predominaron en la época, el Presidente Durán Ballén presentó un proyecto de ley para aumentar la competitividad de Ecuador en la industria petrolera global, reemplazando el modelo del contrato de servicios por aquella del contrato de participación, en que el contratista recibe una participación en la producción del petróleo ${ }^{3}$.

2. Véase S. PAUSHOK, CJSC, Golden East Co. v. the Government of Mongolia, Decisión sobre Jurisdicción y Responsabilidad, 28/04/2011.

3. CIADI, Burlington Resources Inc c. República del Ecuador. Decisión sobre la Jurisdicción del Tribunal Arbitral y Decisión sobre Responsabilidad, Caso No. ARB/08/5, 2/06/2010 y 14/12/2012. CIADI, Perenco Ecuador LTD c. La Republica del Ecuador, Decisión sobre Las Cuestiones Pendientes Relativas a la Jurisdicción y Sobre la Responsabilidad, Caso No. ARB 08/06, 12/09/2014. 
Burlington y Perenco adquirieron en los años 2001 y 2003 los derechos de exploración y explotación de los Bloques 7 y 21 . Asimismo, cabe mencionar, que si bien Burlington también adquirió derechos sobre los bloques 23 y 24, los reclamos correspondientes a esos bloques fueron abandonados en el arbitraje y por lo tanto su análisis excede a este comentario. Por su parte Perenco, una empresa controlada por capitales franceses, era el socio mayoritario del consorcio y el operador de los bloques, mientras que Burlington era el socio minoritario poseyendo el $42,5 \%$ en el Bloque 7 y $46,25 \%$ en el Bloque 21.

A partir del año 2002, el precio del petróleo comenzó a aumentar. Para el año 2008, el precio del barril se encontraba a más de USD 100 y muy por encima del precio de los precios internacionales que existían en 2001 y 2003 cuando se hicieron las inversiones ${ }^{4}$. Ante esta situación, el Presidente ecuatoriano Palacio (2006) presentó un proyecto de Ley al Congreso donde propuso una participación adicional a favor del Estado de "al menos el 50\% de los llamados ingresos extraordinarios", es decir, ingresos derivados del precio del petróleo que superen el precio del petróleo vigente al celebrar los Contratos de Participación ${ }^{5}$. La ley se promulgó como la Ley 42. En 2007, el nuevo Presidente Rafael Correa emitió el Decreto 662, el cual incrementó la alícuota impositiva de la Ley 42 del $50 \%$ al 99\% ${ }^{6}$, probablemente para forzar a las empresas contratistas a aceptar un marco regulatorio distinto en el cual se previera una mayor participación para el Estado.

A diferencia de otros momentos históricos anteriores, el cambio del marco regulatorio y la pretendida participación del Estado en las ganancias extraordinarias producto del incremento en el precio del petróleo, se encontró con Tratados Bilaterales de Protección de Inversiones (TBIs) en vigencia y con una nutrida casuística que desde principios del siglo ha venido acumulando un número considerable de decisiones que sancionan el cambio regulatorio.

\footnotetext{
4. CIADI, Burlington Resources, N. 3, párrs. 23-24.

5. Ídem, párr. 9.

6. Ídem, párr. 35, CIADI, Perenco. N. 3 párrs. 88 y 109.
} 
Dos TBIs son relevantes para este estudio; (a) el "Tratado entre Estados Unidos y Ecuador sobre Promoción y Protección Recíproca de Inversiones" ("TBI de Ecuador con los Estados Unidos") ${ }^{7}$, invocado por Burlington, y (b) el "Convenio entre el Gobierno de la República Francesa y el Gobierno de la República de Ecuador para la Promoción y Protección Recíproca de Inversiones" ("TBI con Francia") ${ }^{8}$. Si bien Perenco es una sociedad constituida conforme a las leyes de la Mancomunidad de las Bahamas, es parte del Grupo de Empresas Perenco, fundado por el difunto Hubert Perrodo, de nacionalidad francesa, quien controlaba el grupo empresario con una participación del $92,9 \%$.

Como veremos, la invocación de estos dos distintos Tratados tendrán consecuencias trascendentales para la jurisdicción de los respectivos Tribunales, pues el TBI con Estados Unidos excluye en su Artículo X a todo acto impositivo del gobierno de Ecuador, a excepción de que constituya una expropiación. ${ }^{9}$ Mientras que el TBI con Francia no excluye cuestiones impositivas de la jurisdicción del Tribunal arbitral. Por ello el Tribunal del caso Burlington determinó que siendo la Ley 42 una disposición impositiva, su jurisdicción sólo alcanzaba los actos de Ecuador si estos constituían una expropiación, excluyendo de su jurisdicción los reclamos por falta de trato justo y equitativo, trato arbitrario o falta de protección y seguridad plena.

La invocación de los diferentes Tratados también aparejó un distinto tratamiento de los reclamos sustanciales impetrados por las Demandantes:

7. Tratado entre Estados Unidos y Ecuador sobre Promoción y Protección Recíproca de Inversiones. El tratado fue adoptado el 27 de Agosto de 1993 en Washington DC, y entro en vigor el 11 de Mayo de 1997, disponible en <http://www.sice.oas.org/bits/usaec_s.asp>.

8. Convenio entre el Gobierno de la República Francesa y el Gobierno de la República de Ecuador para la Promoción y Protección Recíproca de Inversiones. El tratado fue suscrito entre Francia y Ecuador el 7 de Septiembre de 1994.

9. Tratado entre Estados Unidos y Ecuador sobre Promoción y Protección Recíproca de Inversiones, N. 7. clausula X(1) del TBI "En lo relativo a sus normas tributarias, cada Parte deberá esforzarse por actuar justa y equitativamente en el trato de las inversiones de los nacionales y las sociedades de la otra Parte. 2. No obstante, las disposiciones del presente Tratado, especialmente de los Artículos VI y VII del mismo, se aplicarán a cuestiones tributarias solamente con respecto a: a) La expropiación, de conformidad con el Artículo III; [...]”. 
1. Como se vio anteriormente, el reclamo de Burlington quedó limitado al reclamo expropiatorio, pues los reclamos fundados en el tratamiento (trato justo y equitativo, trato nacional y protección y seguridad plena) quedaron fuera del reclamo por efecto del Artículo X del Tratado entre Ecuador y los Estados Unidos, el cual establece que las cuestiones impositivas quedan fueran de la jurisdicción del Tribunal.

2. El Tribunal en Perenco si analizó cado uno de los estándares de tratamiento sustancial de la inversión, concluyendo que: (i) la Ley 42 con una alícuota del 50\% no violó el trato justo y equitativo, porque no hubo ninguna expectativa legítima que haya sido vulnerada, pues un inversor sofisticado como Perenco debía saber que el cambio fundamental de circunstancias, operado por el incremento extraordinario del precio del petróleo, requeriría un ajuste del contrato. No obstante, el Tribunal también determinó que la Ley 42 con una alícuota del $90 \%$, sí era violatorio del estándar del trato justo y equitativo.

3. En relación a la existencia de una expropiación indirecta, las Demandantes sostuvieron, en ambos procedimientos, que el efecto cumulativo de las medidas de Ecuador, comenzando con la promulgación de la Ley 42 (que esencialmente los privó de la oportunidad de obtener ganancias), la aplicación de la Ley 42 a través del embargo y la venta del crudo de petróleo (Procedimiento de Coactiva), la toma física de control de los Bloques y la declaración unilateral de caducidad, resultaron en una incautación total de sus activos $^{10}$. Argumentando, asimismo, que Ecuador no presentó ningún justificativo de necesidad pública para sus acciones y no pagó ni ofreció pagar ninguna compensación.

4. Efectos Expropiatorios de la Ley 42: Tanto en relación a la alícuota del $42 \%$ y del $99 \%$. Para el Tribunal en Burlington la medida no fue expropiatoria. Según el Tribunal, el factor

10. CIADI, Burlington Resources, N. 3, párr. 67. Perenco N. 3, párr. 256. 
más importante para distinguir entre tributación legítima y confiscatoria es el efecto de la misma ${ }^{11}$. Para que exista expropiación el efecto debe ser la privación plena de un derecho contractual, no reparada y sustancial. En este caso el Tribunal consideró que los derechos de Burlington no fueron sustancialmente privados de valor ${ }^{12}$. La Ley 42 sea con una alícuota del 50\% o del $99 \%$ no afectó la economía de los $\mathrm{CP}$ y por lo tanto no es expropiatoria ${ }^{13}$. El Tribunal en Perenco resolvió que: (i) La Ley 42 con una alícuota del 50\% y con una alícuota del $90 \%$ no privó sustancialmente a Perenco de la inversión ni del control sobre la misma y por lo tanto, no fue una medida equivalente a la expropiación indirecta $^{14}$.

5. Efectos Expropiatorios de los Juicios Coactivos: El Tribunal en el caso Burlington resolvió que (i) respecto del juicio de coactivas Burlington no fue privado sustancialmente de los derechos de su inversión, y por lo tanto no fue expropiatorio ${ }^{15}$; (ii) Ecuador estaba ejecutando la suma total de los impuestos adeudados en 2008 por Burlington conforme a la Ley 42. Una vez que estos impuestos adeudados hubieran sido pagados, Burlington hubiera continuado percibiendo su participación en la producción de petróleo, al valor económico disminuido por cualquier nuevo impuesto de la Ley $42^{16}$. Para el Tribunal en el caso Perenco la ejecución de las coactivas no constituyó una expropiación a la inversión, aunque como se señaló anteriormente sí constituyó un incumplimiento de la obligación de conferir un trato justo y equitativo ${ }^{17}$.

11. CIADI, Burlington Resources, N. 3, párr. 395.

12. Ídem, párr. 388.

13. El Tribunal también razonó que inclusive en el supuesto caso de que hubiera afectado la economía de los CP, tampoco sería sinónimo de incumplimiento automático del TBI con Estados Unidos, sino un mero incumplimiento contractual -que no puede ser equiparado a la confiscación de una propiedad-. Ídem, párr. 383 y 384.

14. CIADI, Perenco, N. 3, párrs. 680, 681 y 684.

15. CIADI, Burlington Resources, N. 3, párr. 485

16. Ídem, párr. 479.

17. CIADI, Perenco, N. 3, párr. 703. 
6. Efectos Expropiatorios de la ocupación física de los Bloques 7 y 21 por Ecuador: El Tribunal del caso Burlington encontró que una vez que Ecuador toma posesión física de los bloques debía indemnizar a Burlington, y al no hacerlo lo convierte en una expropiación ilegítima ${ }^{18}$, exceptuando los límites establecidos por el Artículo X del Tratado y permitiendo así a la jurisdicción del Tribunal para juzgar sobre esos efectos ${ }^{19}$. El Tribunal, contrariamente a Burlington, resolvió; (i) que cuando el Consorcio anunció su intención de suspender las operaciones, había razones justificadas y válidas para que Ecuador interviniera con el fin de operar los Bloques, para así garantizar su continuidad y mantener su productividad; y (ii) que Ecuador tenía el derecho a operar y mantener los Bloques luego de que el Consorcio se había retirado. Esta intervención -que no interfirió con el derecho del Consorcio de administrar y controlar los Bloques porque el Consorcio había abandonado dicho derecho en forma voluntaria y temporal no constituyó una expropiación $^{20}$.

7. Efectos Expropiatorios de Decreto de Caducidad: El Tribunal en Burlington resolvió que la terminación de los CP de los Bloques 7 y 21 por medio del procedimiento de caducidad meramente formalizó una situación imperante, pero es irrelevante a los fines del análisis de la expropiación. Como resultado, el Tribunal omitió la revisión de las alegaciones y los argumentos específicos efectuados en relación con la caducidad ${ }^{21}$. La decisión del Tribunal en Perenco es diametralmente opuesta. El Tribunal resolvió que (i) si bien Ecuador tenía el derecho a intervenir y operar los bloques no

18. Ídem, párr. 543.

19. El Tribunal en Burlington considera que la suspensión que hizo de la operación en los Bloques estaba legalmente justificada por el derecho ecuatoriano y el derecho internacional, y que Ecuador expropió ilegítimamente su inversión al ocuparlos físicamente. Ecuador considera que la suspensión por parte de Burlington no estaba amparada por el derecho ecuatoriano, derecho internacional ni por los $\mathrm{CP}, \mathrm{y}$ que fue una puesta en escena para obligarlo a intervenir y aparentar una expropiación indirecta. La ley de Hidrocarburos de Ecuador en su Artículo 74 faculta al Ministerio de Recursos Naturales no Renovables a terminar un contrato decretando su caducidad cuando se suspendieren las operaciones de explotación por más de 30 días sin justa causa.

20. CIADI, Perenco, N. 3, párr. 705.

21. CIADI, Burlington Resources, N. 3, párr. 536. 
estaba obligado a dar por extinguidos los CP mediante una declaración de caducidad. El Ministerio está facultado para declarar la caducidad de los contratos ante alguna de las circunstancias específicas de la Ley de Hidrocarburos de Ecuador, pero no está obligado a hacerlo22; (ii) dicha potestad debería haberse ejercido en pos de evitar el proceso de caducidad mientras se esperaba una declaración sobre los derechos y las obligaciones en el proceso arbitral; y por ello (iii) a partir de la fecha de la declaración de la caducidad, momento en que se extinguieron los derechos del Consorcio, Ecuador expropió los derechos contractuales de Perenco en violación del Artículo VI del Tratado.

Finalmente, es importante señalar que el Tribunal en Perenco entendió que tenía jurisdicción para entender sobre los reclamos contractuales, en la medida que el CIADI era también la jurisdicción pactada en el contrato de inversión. El Tribunal resolvió que (i) respecto de la Ley 42 con una alícuota del 50\%, la cual posiblemente cambió la economía del $\mathrm{CP}$, no violó el $\mathrm{CP}$ porque un inversor sofisticado como Perenco debía saber que el cambio de circunstancias requeriría un ajuste del contrato; y, (ii) respecto de la Ley 42 con una alícuota del 90\% existió un incumplimiento contractual del $\mathrm{CP}^{23}$. 


\begin{tabular}{|c|c|c|}
\hline Medidas de Ecuador & Burlington & Perenco \\
\hline $\begin{array}{l}\text { Las Medidas Impositi- } \\
\text { vas están exceptuadas } \\
\text { de la Jurisdicción del } \\
\text { Tribunal excepto en el } \\
\text { caso de Expropiación }\end{array}$ & $\begin{array}{l}\text { Si. Por efecto del Artí- } \\
\text { culo X del Tratado } \\
\text { entre Ecuador y los } \\
\text { Estados Unidos. }\end{array}$ & $\begin{array}{l}\text { No. No existe similar } \\
\text { norma similar en el } \\
\text { Tratado entre Ecuador } \\
\text { y Francia. }\end{array}$ \\
\hline $\begin{array}{l}\text { Efectos Expropiatorios } \\
\text { de la Ley } 42 \text { con una } \\
\text { alícuota del } 50 \%\end{array}$ & No. & No. \\
\hline $\begin{array}{l}\text { Efectos Expropiatorios } \\
\text { de la Ley } 42 \text { con una } \\
\text { alícuota del } 99 \%\end{array}$ & No. & No. \\
\hline $\begin{array}{l}\text { Efectos Expropiatorios } \\
\text { de los Juicios Coacti- } \\
\text { vos. }\end{array}$ & No. & $\begin{array}{l}\text { No. Sin embargo, en- } \\
\text { contró que los juicios } \\
\text { coactivos si violaban } \\
\text { el estándar de trato } \\
\text { justo y equitativo. }\end{array}$ \\
\hline $\begin{array}{l}\text { Efectos Expropiatorios } \\
\text { de la Ocupación Física } \\
\text { de los Bloques } 7 \text { y } 21 .\end{array}$ & Si. & No. \\
\hline $\begin{array}{l}\text { Efectos Expropiatorios } \\
\text { del Decreto de Cadu- } \\
\text { cidad }\end{array}$ & No. & Si. \\
\hline $\begin{array}{l}\text { Efectos sobre el Trato } \\
\text { Justo y Equitativo de } \\
\text { la Ley } 42 \text { con una alí- } \\
\text { cuota del } 50 \%\end{array}$ & $\begin{array}{l}\text { No se expide al haber } \\
\text { declarado la inexisten- } \\
\text { cia de jurisdicción por } \\
\text { efecto del Artículo X } \\
\text { del Tratado. }\end{array}$ & No. \\
\hline $\begin{array}{l}\text { Efectos sobre el Trato } \\
\text { Justo y Equitativo de } \\
\text { la Ley } 42 \text { con una alí- } \\
\text { cuota del } 99 \%\end{array}$ & $\begin{array}{l}\text { No se expide al haber } \\
\text { declarado la inexisten- } \\
\text { cia de jurisdicción por } \\
\text { efecto del Artículo X } \\
\text { del Tratado. }\end{array}$ & $\begin{array}{l}\text { Si viola el estándar de } \\
\text { Trato Justo y Equita- } \\
\text { tivo. }\end{array}$ \\
\hline
\end{tabular}




\section{La Fisonomía de los Procedimientos Paralelos}

Nada impide que en determinadas circunstancias, una misma controversia desencadene dos o más procedimientos. Ello puede suceder en un procedimiento (arbitral) de inversión sino también en la justicia doméstica de los distintos países. Sin embargo, todos los sistemas jurídicos tienen una forma de evitar la sustanciación paralela de procedimientos, obligando a alguno de los magistrados a declinar su jurisdicción.

La mayoría de los Tratados de Protección de Inversiones, salvo honrosas excepciones que confirman la regla [y que analizaremos a continuación], no prevén una solución para procedimientos arbitrales paralelos. Inclusive, las normas de estos Tratados -a través de su letra o de su silencio- parecen promoverlo. Asimismo, las normas procesales (sean las Reglas de Procedimiento del CIADI o los reglamentos de la CNUDMI) mantienen absoluto silencio al respecto y los Tribunales internacionales no se han mostrado dispuestos a considerar que ante el silencio de los Tratados era de aplicación el Derecho Internacional General que disponía de normas al respecto.

Inclusive asumiendo el riesgo de olvidar alguna de las causales, y apartándonos parcialmente de las consideraciones realizadas por CREMADES y MADALENA, las principales razones por las que se presentan procedimientos paralelos en el arbitraje internacional de inversiones son las siguientes:

(a) La proliferación de Tratados de Inversión

(b) Los reclamos que el inversor puede realizar frente a los Tribunales domésticos del país receptor de la inversión

(c) Los reclamos que el inversor puede realizar bajo el contrato

(d) Las Acciones Indirectas

(e) La carencia de un sistema efectivo y obligatorio de acumulación de procedimientos. 
Sin embargo, y por las razones que explicaremos, dos de estas causales explican la casi totalidad de la casuística: las acciones indirectas y la inexistencia de un sistema de acumulación de procedimientos. Las demás causales pueden fácilmente ser excluidas, y así ha ocurrido por efecto de las decisiones de los Tribunales.

(a) Como explicaremos a continuación, la proliferación de Tratados de Inversión no es por sí misma la causal de la existencia de procedimientos paralelos. La proliferación de tratados requiere una causa adicional, especialmente aquella que denominamos acciones indirectas para constituirse en la fuente de procedimientos paralelos ${ }^{24}$.

(b) El problema de la duplicidad de reclamos frente a los Tribunales domésticos del país inversor y el inicio de un arbitraje de inversión, ha sido resuelto a través de la norma existente en un número considerable de tratados y que ha recibido coloquialmente el nombre de fork in the road. Es decir, habiendo optado el inversor por iniciar un procedimiento judicial, ello conlleva automáticamente el abandono de la vía arbitral. Asimismo, es necesario considerar que pocos inversores intentarán recurrir a los Tribunales domésticos pues la existencia misma de este sistema arbitral se fundamenta en una desconfianza en los Tribunales locales.

(c) Los reclamos paralelos bajo el Contrato o bajo el Tratado, hace tiempo que han dejado de ser una preocupación para los Tribunales. Aunque el reclamo bajo el Tratado surja en relación a una actividad contractual, la base [entiéndase fuente, como causa] del reclamo debe encontrarse en el Tratado, es decir, la norma violada tiene que ser una norma de derecho público internacional contenida en el Tratado ${ }^{25}$. Por supuesto, los reclamos paralelos pueden coexistir, pero su contenido, el tipo

24. Hay sí un fenómeno que pocas veces se ha planteado: que una parte Demandante invoque más de un Tratado, al poder esa parte reclamar más de una nacionalidad. No analizaremos aquí esa conducta, sólo diremos que sin las denominadas acciones indirectas, tampoco este fenómeno se presentaría.

25. Excepción a este principio puede darse en el caso de que exista en el tratado respectivo una cláusula paraguas. Sin embargo, la interpretación de los efectos de la cláusula paraguas no es unívoca, y por lo tanto omitiremos esa temática en el presente análisis. 
de norma y las consecuencias jurídicas (especialmente la valoración de los daños) serán enteramente diversas.

Un fenómeno aparte, a los fines de la causación de procedimientos paralelos y potencialmente sentencias contradictorias son las acciones indirectas y la carencia de un sistema efectivo de acumulación de procedimientos:

\section{Acciones Indirectas}

Las acciones indirectas son los reclamos presentados por los distintos accionistas que constituyen un consorcio o por los individuos que realizan una inversión en conjunto y que reclaman por su cuota parte. De esta manera, y respecto a un solo conjunto de circunstancias fácticas, se presentan reclamos autónomos generando una pléyade de Tribunales arbitrales dispuestos, cada uno, a hacer escuchar su propia opinión sobre los hechos o el derecho aplicable. Muchos de los casos más notorios y que se citan frecuentemente como los ejemplos más ominosos de procedimientos arbitrales surgen de esta circunstancia. Burlington y Perenco (contra la República del Ecuador), Lauder y CME (contra la República Checa), CMS y LGE (contra la República Argentina), pertenecen a esta categoría ${ }^{26}$.

\section{Inexistencia de un Mecanismo de Acumulación de Procedimientos}

Con la distinguida excepción del NAFTA, la Acumulación de Procedimientos no se encuentra prevista en la mayoría de los Tratados bilaterales de inversión y prácticamente ausente en las principales normas de procedimientos aplicables a los procedimientos de inversión. Por ello, y especialmente en el caso que medidas del Estado que afectan a un cúmulo de inversores extranjeros, como ocurriera con la Crisis Económica y Social en Argentina (2001) o con la Ley 42, como en el caso bajo estudio, la

26. Para mayor abundamiento sobre esta cuestión véase G. BotTINI, "Indirect Claims under the ICSID Convention”, Penn. Journal of International Law, Vol. 29, 2008. 
falta de una norma clara de acumulación de procedimientos y que no requiera de la voluntad de las Partes, arroja necesariamente [por la autonomía de los Tribunales arbitrales y la individualidad de sus miembros] resultados contradictorios que atentan contra la credibilidad del sistema de solución de controversias $^{27}$.

El arbitraje internacional fue pensado como un medio para resolver asuntos entre comerciantes y hacerlo en forma rápida y confidencial en modo de no afectar una relación que en la medida de lo posible debe continuar. Por el contrario, un arbitraje de inversión plantea un escenario en que la relación entre el Estado y el inversor ha llegado a tal deterioro que el inversor ha sido objeto de un alegado tratamiento abusivo por parte del Estado (violación de alguno de los estándares de tratamiento) o el Estado ha tomado la propiedad sobre los bienes objeto de la disputa (expropiación) que conlleva la violación de estándares mínimos de civilidad.

En el arbitraje [comercial] internacional puede haber una decisión injusta o inclusive una decisión carente de fundamentos porque los valores inmediatos que persigue son los de la resolución rápida y efectiva de los conflictos. En el arbitraje de inversión, por el contrario, y dada la presencia del Estado como uno de los contendientes, la protección de la cosa pública y el bien común de los ciudadanos en el corazón de la disputa, se exige que la decisión se encuentre debidamente fundamentada y alcance los más altos estándares de justicia, objetivos que la celeridad del arbitraje (comercial) internacional no necesariamente persigue.

Por ello, las decisiones contradictorias ante situaciones fácticas y jurídicas similares que generadas por los procedimientos paralelos acarrean la pregunta necesaria de si el actual mecanismo de solución de disputas no requiere de urgentes reformas para realmente satisfacer a sus usuarios y aquellos terceros [como

27. Véase B. Cremades e I. Madalena, N. 1, p. 80. 
La Historia de dos Ciudades:

Procedimientos Paralelos y Reformas al Arbitraje de Inversión

la sociedad civil] que tienen la expectativa de que la disputa sea resuelta conforme a los más altos estándares de la civilización moderna.

\section{Conclusión}

Las Decisiones arbitrales en los casos Burlington y Perenco ejemplifican las consecuencias negativas que acarrea el hecho de que un mismo escenario fáctico sea analizado por dos o más tribunales actuando en forma totalmente autónoma uno de otro y sin que exista un procedimiento posterior de casación que pueda consensuar ambas decisiones. Dado que estos tribunales están compuestos por distintos individuos, las decisiones sólo pueden ser diferentes, tanto en el resultado económico como en los argumentos legales con los que cada Tribunal justifica sus decisiones.

Al momento en que escribo esta contribución, todavía no se conoce el monto de la indemnización que Ecuador deberá pagar en cada uno de estos arbitrajes, aunque sí sabemos que ha sido condenado en ambos procedimientos. La frialdad de los números nos hará saber en cuánto difieren las decisiones. No obstante, las diferencias en los razonamientos legales ya son patentes. Cada Tribunal fundamenta su decisión, como si ambos hubieran estado juzgando distintos hechos y aplicando distinto derecho, cuando paradójicamente la situación fáctica es idéntica, y más allá del Artículo X del Tratado entre Ecuador y los Estados Unidos, el derecho aplicable es el mismo (concepto de expropiación en el derecho internacional público).

Este distinto tratamiento no solo afecta al Estado sino también al inversor y llena de incertidumbre las relaciones jurídicas. Pareciera difícil que un especialista en la materia, que es consultado por un cliente, sea éste el Estado o el inversor, pueda dar a priori un parecer inequívoco sobre el posible resultado de una disputa que es sometida a su consideración. Al ser el Arbitraje de Inversión un sistema totalmente descentralizado, sólo es posible 
ensayar teorías a partir de tendencias, pero claramente no es posible determinar la existencia de una jurisprudencia.

Esta incoherencia (en los resultados) que observamos crea incertidumbre y ofrece un perfil del sistema de inversión susceptible de fácil crítica por aquellos detractores del sistema. Asimismo, la parte que resulte perdidosa quedará con el sabor amargo de una decisión que no necesariamente responde al valor justicia sino a la conformación del Tribunal arbitral lo cual plantea el gran interrogante de sí es el arbitraje (tal como lo conocemos hoy) la forma más apta para resolver disputas que requieren justicia y una detallada fundamentación de la decisión. Tampoco debe olvidarse que estas decisiones son definitivas y que los remedios que existen hacen sólo a la integridad del procedimiento (aunque normalmente se abusen en la búsqueda quimérica de revertir el resultado). 